
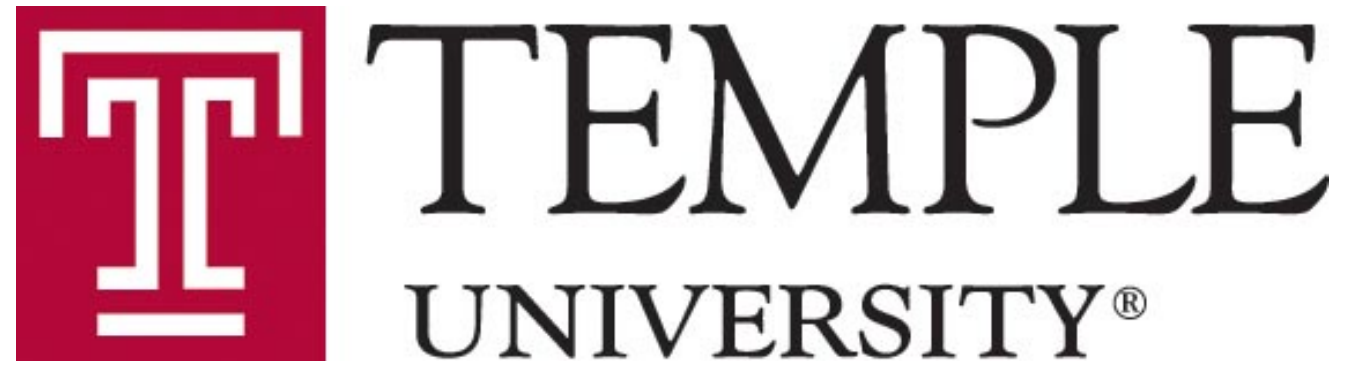

\title{
Structural Sign Patterns and Reduced Form Restrictions
}

\author{
by \\ Andrew J. Buck \\ George M. Lady \\ Department of Economics \\ DETU Working Paper 11-02 \\ June 2011
}

1301 Cecil B. Moore Avenue, Philadelphia, PA 19122 http://www.cla.temple.edu/economics/ 


\title{
Structural Sign Patterns and Reduced Form Restrictions \\ By \\ Andrew J. Buck and George M. Lady ${ }^{1}$
}

\begin{abstract}
This paper reconsiders the degree to which the sign patterns of hypothesized structural arrays limit the possible outcomes for the sign pattern of the corresponding estimated reduced form. To now, the conditions under which any such restrictions would apply were believed to be very narrow, are rarely found to apply, and are virtually never investigated. As a result, current practice does not test the structural hypothesis in terms of the outcome of the estimated reduced form in terms of permissible reduced form sign patterns alone. This paper shows that such tests are always possible. Namely, that the sign patterns of the hypothesized structural arrays always limit the sign patterns that can be consistently taken on by the estimated reduced form. Given this, it is always possible to falsify a structural hypothesis based only upon the sign pattern proposed. Necessary conditions, algorithmic principles, and examples are provided to illustrate the analytic principle and the means of its application.
\end{abstract}

JEL Classification: C15, C18, C51, C52

Key Words: Qualitative analysis, structural form, reduced form, identification, model falsification

\footnotetext{
${ }^{1}$ The authors are Professors of Economics at Temple University, Philadelphia, PA 19025. Contact: George M.
} Lady:gmlady@ix.netcom.com or Andrew J. Buck: buck@temple.edu. 


\section{Structural Sign Patterns and Reduced Form Restrictions}

I. Background. It is common practice in economics to represent aspects of the way the economy works by a mathematical "model" comprised of a system of simultaneous equations:

$$
\mathrm{f}^{\mathrm{i}}(\mathrm{Y}, \mathrm{Z})=0, i=1,2, \ldots, n,
$$

where $\mathrm{Y}$ is an $\mathrm{n}$-vector of endogenous variables and $\mathrm{Z}$ is an $\mathrm{m}$-vector of exogenous variables. Examples include the first order conditions for an (often constrained) optimization problem, a set of excess demand functions associated with multiple markets, or behavioral and accounting equations describing features of (or its aggregative entirety) the macro-economy.

The solutions to such systems, per se, are austere in terms of the hypotheses that they present that can be tested with data. Instead, richer hypotheses are derived from a comparative statics analysis of the system. Such analyses study the impacts of changes in the entries of $\mathrm{Z}$ upon the solution values of the entries of Y via a linear system of differentials,

$$
\sum_{j=1}^{n} \frac{\partial f^{i}}{\partial y_{j}} d y_{j}+\sum_{k=1}^{m} \frac{\partial f^{i}}{\partial z_{k}} d z_{k}=0, i=1,2, \ldots, n
$$

When this method is brought to the data, it is not uncommon to assume that the system (2) is (at least locally) linear and, with error terms added, the system (2) is re-expressed as,

$$
\beta Y=\gamma Z+\delta U
$$

where $\beta, \gamma$ and $\delta$ are appropriately dimensioned matrices and $U$ is a matrix of disturbances. ${ }^{2}$ The system (3) is usually called the structural form of the model. Significantly, (3), specifically the

\footnotetext{
${ }^{2}$ For early macro models as proposed by (such as) the Cowles Foundation, $\mathrm{Y}$ was of dimension Gx1, Z was Kx1 and $\mathrm{U}$ was a Gx1 error vector. In that era $\beta$ and $\gamma$ were sparse, appropriately dimensioned matrices of unknowns and $\delta$
} 
arrays $\{\beta, \gamma, \delta\}$, may be taken as the hypothesis that economic theory proposes about the economy.

As a step on the road to evaluating hypotheses about $\beta, \gamma$ and $\delta$, (3) must be manipulated before being taken to the data. The result is,

$$
\mathrm{Y}=\pi \mathrm{Z}+\Psi \mathrm{U}
$$

The system (4) is usually called the reduced form for which $\pi=\beta^{-1} \gamma$. As practiced, $\pi$ and $\Psi$ can be estimated directly and, via identifying restrictions, estimates of $\beta, \gamma$ and $\delta$ are constructed. The hypothesis $\{\beta, \gamma, \delta\}$ in (3) is then tested, after manipulating the outcome of the unrestricted estimation of $\pi$ (or sometimes $\Psi$ as well) in (4), to produce an estimate of the unknowns in (3) and their associated test statistics. However, from the standpoint of economics as a "science," the hypothesis $\{\beta, \gamma, \delta\}$ (presumably) imposes restrictions on the possible outcomes of the estimated $\pi$. If these restrictions are not found to be the case, even before constructing estimates of $\{\beta, \gamma, \delta\}$ from the estimated $\pi$, then the hypothesis (within the limits of the data used) has been "falsified" (Popper (1934/1959)).

An immediate issue is what the restrictions are that an hypothesis $\{\beta, \gamma, \delta\}$ imposes on the outcome of the estimation of $\pi$. This issue has been, and remains, somewhat problematical. The problem was posed over sixty years ago by Samuelson (1947). He first noted that economic theory sometimes only specifies the sign patterns of $\{\beta, \gamma\}{ }^{3}$ Given this, restrictions on $\pi$ (presumably on the signs of its entries) must somehow be found through the algebra involved in $\pi=\beta^{-1} \gamma$, a so-called qualitative analysis. Samuelson thought that it would be very unlikely that restrictions could be found this way. Instead, he proposed other criteria. For optimization problems, the second order conditions for solution can require that certain entries of $\beta^{-1}$ take on specific signs. Similarly, for (say) multimarket equilibria, if the system (3) is assumed to be

was an identity matrix. In the era of structural VARs $\gamma$ was no longer sparse and $\beta$ was identified via restrictions on the covariance matrix of the random vector $U$. In more contemporary work $U$ is a matrix and $\delta$ is no longer the identity matrix. Identification is achieved by zero restrictions in $\{\beta, \gamma, \delta\}$ and on the disturbance covariance matrix.

${ }^{3}$ At this juncture we drop consideration of $\delta$ and $\Psi$ for purposes of expositional simplicity; $\delta$ is now understood to be the identity matrix. The approach we advocate here is fully applicable to the more general case. 
stable, then certain entries of $\beta^{-1}$ must take on specific signs, i.e., the Correspondence Principle. Sometimes invoking functional forms can help. ${ }^{4}$ Samuelson (op. cit.) also noted that the relative size of some entries of $\beta^{-1}$ could be related to the number of constraints imposed in an optimization problem; and later, Samuelson (1960), showed the same result for stable, multimarket equilibria, both as applications of the LeChatelier Principle. ${ }^{5}$

It is a fair question to ask how things have changed since these ideas were first expressed. In spite of Samuelson's pessimism, a literature on the conditions under which a qualitative analysis would be successful did develop. Yet, the conditions are very restrictive and are rarely found to be applicable (or even investigated for that matter), just as Samuelson surmised. ${ }^{6}$ At the same time a very substantial econometrics literature developed around the use of the unrestricted estimated $\pi$ in the derivation of estimates of $\{\beta, \gamma\}$. Identification issues can complicate, or even compromise, this recovery process. And in any event, what is done is not usually presented as a "test" of the hypothesized model in the sense of potential falsification. Specifically, there has not been much attention to the restrictions on $\pi$ due to the proposed hypothesis $\{\beta, \gamma\}$ that are then submitted to econometric analysis. ${ }^{7}$ In sum, there really has not been much progress, or related practice, in testing the hypotheses advanced by economic theory in the sense of a formal investigation of how the structural specification limits the estimated reduced form outcome.

The point of this paper is to propose a method that enables examining the restrictions on $\pi$ imposed by the hypothesis $\{\beta, \gamma\}$ thereby providing the potential for falsifying the model (3) regardless of the numerical outcomes for $\{\beta, \gamma\}$ as derived from the unrestricted estimate of $\pi$. The method of analysis proposed involves the relaxation of a condition traditionally required of qualitative analyses that has little consequence in terms of actual, applied systems (sign nonsingularity is not assumed, see below). It is demonstrated that Samuelson's initial pessimism and

\footnotetext{
${ }^{4}$ E.g., Hicks (1932) assumes the production function is homogeneous of degree one when demonstrating "Marshall's Rules" for the size of factor price elasticities.

${ }^{5}$ Lady and Quirk $(2007,2010)$ showed that the LeChatelier Principle could be established based upon the sign pattern of $\beta$.

${ }^{6}$ Hale, et al (1999) reviews much of this literature. Additional citations are provided in the next section.

${ }^{7}$ Buck and Lady (2005) consider this problem using a traditional qualitative analysis and the stability hypothesis.
} 
the restrictions traditionally required for such an analysis are overdone. In fact, we show that a specification of the sign pattern of the structural arrays always imposes significant limitations on the sign pattern that the associated reduced form array can take on. As a result, the potential for examining model specification is significantly increased, based only upon a qualitative specification of the structural form.

In the next section a brief review of traditional qualitative methods is presented. Some of the methods traditionally used can be useful in the more general method presented here. In section III the enhanced method for conducting a qualitative analysis is presented. Examples are given in section IV. The last section provides a summary of results. Derivations associated with the examples are provided in an appendix.

II. Qualitative Analysis. For the rest of the paper, the system (3) will be simplified by the assumption that $\gamma=\mathrm{I}$, so that $\pi=\beta^{-1}$. This assumption is typical of the literature on qualitative analysis, but is not entirely without consequence. Buck and Lady (2005) provide examples of a traditional analysis without this simplification. In addition, it will be assumed that the matrix $\beta$ is irreducible, i.e., no entry of $\beta^{-1}$ must be zero. Finally, it will be assumed that no entry of $\beta^{-1}$ is otherwise equal to zero, i.e., no array corresponding to a cofactor is singular. These last two assumptions are convenient and the analysis we develop can be readily extended to exclude them. ${ }^{8}$

The problem at issue is this: Given $\operatorname{sgn} \beta$, can it be shown that some, if not all, entries of sgn $\beta^{-1}$ have certain signs, independent of the magnitudes of the entries of $\beta$.

\footnotetext{
${ }^{8}$ A quick take on limiting the analysis to cases for which $\gamma=$ I might be proposed to imply that the estimate of $\beta$ could "simply" be derived by inverting the reduced form. Consistency of the sign patterns involved could be determined by comparing the sign pattern of this result with that hypothesized for $\beta$. Doing this does not account for the identification problem if the hypothesized $\beta$ contains zero entries, as it typically would. If $\beta$ contains zero entries, then some version of multi-stage least squares would be used to recover the estimated $\beta$ from the estimated reduced form. The possible approaches need not be in agreement as to the sign pattern of the recovered $\beta$. Further, under current practice, they would be undertaken even if the sign pattern of the estimated reduced form was an impossible outcome, given the hypothesized sign pattern for $\beta$ or even only its hypothesized zeros, i.e., since no test for these circumstances is made.
} 
Lancaster (1962) initiated a literature on this issue by proposing that the answer is "yes" if and only if $\beta$ can be manipulated into a particular form. ${ }^{9}$ Gorman (1964) showed that Lancaster's proposed form was sufficient, but not necessary. Somewhat different methods were developed using the theory of signed, directed graphs. ${ }^{10}$ A key condition is to show the necessary and sufficient conditions on sgn $\beta$ such that det $\beta \neq 0$ independent of the magnitudes of $\beta$ 's entries. If these conditions are satisfied, then $\beta$ is called "sign non-singular" or "qualitatively invertible" and at least some entries of $\operatorname{sgn} \beta^{-1}$ must have particular signs. ${ }^{11}$ These conditions are presented in Bassett, Maybee and Quirk (1968). The graph theoretic basis and reasoning for the conditions are given below in the Appendix. In summary, cycles of inference are identified with each evaluated as the product of associated entries of $\beta$. The fundamental theorem of qualitative analysis is then:

Theorem (Sign Non-Singularity, Bassett, Maybee and Quirk (1968)): Let $\beta$ be an n $\mathrm{n}$ irreducible matrix in standard form, i.e., transformed such that $\beta_{\mathrm{ii}}<0$ for all i. $\beta$ is sign nonsingular, i.e., sgn det $\beta=(-1)^{\mathrm{n}}$ independent of the magnitudes of the entries of $\beta$, if and only if all of $\beta$ 's cycles of inference have negative values.

The conditions for sign non-singularity are extremely restrictive and are seldom satisfied by any matrix associated with applied models. ${ }^{12}$ Accordingly, we do not assume or require sign nonsingularity. Instead, we assume that $\beta$ is quantitatively non-singular. In effect this removes any restriction upon the array assumed for the structural model, since applied models are not singular as a practical matter. Given this, the conditions we develop in the next section can be applied to any model for which $\beta$ is numerically non-singular.

\footnotetext{
${ }^{9}$ The (upper) Hessenberg Form, e.g., Horn and Johnson (1985), p. 28.

10 This approach is a useful way to express the inference structure of a system of equations and has reappeared in recent years in (such as) Awokuse and Bessler (2003) for the circumstance that the approach provides an ordering of the equations.

${ }^{11}$ Lady (1983) showed that for $\beta$ arranged such that $\beta_{\mathrm{ii}} \neq 0$ for all $\mathrm{i}$ and sign non-singular, then for $\beta_{\mathrm{ij}} \neq 0$, sgn $\beta_{\mathrm{ji}}{ }^{-1}=$ $\operatorname{sgn} \beta_{\mathrm{ij}}$, independent of the magnitudes of the nonzero entries of $\beta$.

12 As noted, this was Samuelson's speculation and it was recognized by the developers of a traditional, qualitative analysis. A variety of algorithmic principles were developed to deal with the need for additional information, e.g., Ritschard (1983), Maybee and Weiner (1988), Gillen and Guccione (1990), and Lady (2000). Even so, the point of the analysis remained that of signing individual entries of $\beta^{-1}$. As shown below, we demonstrate that the analysis can be taken to involve limits on the entire sign pattern of $\beta^{-1}$, even though no individual entries can be signed.
} 
III. The Qualitative Inverse. The starting point for the analysis is the concept of the qualitative inverse.

Definition (qualitative inverse): Let $\beta$ be an $\mathrm{n} \times \mathrm{n}$ irreducible matrix with sign pattern sgn $\boldsymbol{\beta}$. Sgn $\pi$ is a qualitative inverse of $\beta$ if and only if there exist magnitudes for the entries of $\beta$, consistent with $\operatorname{sgn} \boldsymbol{\beta}$, such that $\operatorname{sgn} \beta^{-1}=\operatorname{sgn} \pi$.

From the standpoint of falsifying an hypothesized $\operatorname{sgn} \beta$ by estimating $\pi$, the hypothesized $\operatorname{sgn} \beta$ is consistent with the data only if $\operatorname{sgn} \widehat{\boldsymbol{\pi}}$ is a qualitative inverse of $\beta .{ }^{13}$ Otherwise, within the limits of the data used, the hypothesis is falsified. This criterion picks up all instances in which a traditional qualitative analysis proceeds with $\beta$ sign non-singular. The point of the analysis here is that any hypothesized sgn $\beta$ imposes restrictions on the sign pattern that the estimated $\pi$ can take on; and, given this, any hypothesized $\beta$ can be potentially falsified. The issue thus becomes that of determining if a given sgn $\widehat{\boldsymbol{\pi}}$ (the sign pattern of an estimate of $\pi$ ) is, or is not, a qualitative inverse of an hypothesized $\beta .^{14}$

Let $B=\left[B_{i j}\right]$ be the adjoint of $\beta$. For a given sgn $\widehat{\pi}$, consider the system $(s)$ of inequalities as written out symbolically as the expansions of $\beta$ 's cofactors and determinant,

$$
\operatorname{sgn} B=\operatorname{sgn} \widehat{\boldsymbol{\pi}} \text {, and det } \beta>0 \text {; and/or, } \operatorname{sgn} B=-\operatorname{sgn} \widehat{\boldsymbol{\pi}} \text {, and } \operatorname{det} \beta<0 \text {. (5) }
$$

It is immediate that a given sgn $\widehat{\boldsymbol{\pi}}$ is a qualitative inverse of a proposed $\beta$ if and only if at least one of the systems (5) has a solution. The problem is finding (algorithmically) if any solutions exist. One way is Monte Carlo. The absolute values of the nonzero entries of $\beta$ can be sampled based upon distributional rules, the appropriate sign pattern imposed, the consequent array inverted, and the sign pattern found noted and compared to the given sgn $\widehat{\boldsymbol{\pi}}$. The sampling can

\footnotetext{
${ }^{13}$ Sufficiency might be questioned if it could be shown that the values required for the entries of $\beta$ so as to generate the sign pattern of the estimated reduced form cannot be supported by the theory, i.e., that the theory requires quantitative properties of $\beta$ in addition to its sign pattern.

${ }^{\mathbf{1 4}}$ Even if $\gamma \neq \mathrm{I}$, the estimated $\pi$ would inherit restrictions due to the restrictions on $\beta^{-1}$.
} 
be done over and over to see if the given sgn $\widehat{\boldsymbol{\pi}}$ turns up. If it does, then the given sgn $\widehat{\boldsymbol{\pi}}$ is a qualitative inverse of the proposed sgn $\beta$.

But suppose the given sgn $\widehat{\boldsymbol{\pi}}$ does not appear in the Monte Carlo. In principle, if enough sample $\beta$ are constructed, inverted, and the results compared to a proposed sgn $\widehat{\pi}$, the likelihood of missing sgn $\widehat{\boldsymbol{\pi}}$, assuming it is a qualitative inverse of $\beta$, can be made vanishingly small. Still, for large arrays particularly, "enough" might be quite a few. And even so, if sgn $\widehat{\boldsymbol{\pi}}$ is not found, there would be the nagging thought that the sample is too small. Although it must be said that under these circumstances the proposed hypothesis is at best calling for an unlikely outcome, it nevertheless might be consistent with the data and the Monte Carlo sample is just failing to show it. Better to have some analytic capacity for reaching a judgment before facing up to the analytic burden of determining whether or not (5) has a solution. And, additionally, it needs (here) to be shown that any proposed sgn $\beta$ necessarily restricts the sgn $\pi$ that can be its qualitative inverses; and hence, any proposed sgn $\beta$ can be potentially falsified.

Restrictions that sgn $\beta$ imposes upon sgn $\pi$ can be readily developed. Consider that if and only if $\operatorname{sgn} \pi$ is a qualitative inverse of $\beta$, then there exist magnitudes for the entries of each array such that $\beta \pi=\mathrm{I}$ and $\pi \beta=\mathrm{I}$. For this to be possible, the terms in the sums given in (6) below must have positive, or positive and negative, terms as appropriate.

$$
\sum_{k=1}^{n} \beta_{i k} \pi_{k j} \text { and } \sum_{k=1}^{n} \pi_{i k} \beta_{k j}, i, j=1,2, \ldots, n \text {. }
$$

Call $\operatorname{sgn} \pi$ "id-consistent" with sgn $\beta$ if the outcomes $\beta \pi=\mathrm{I}$ and $\pi \beta=\mathrm{I}$ are not impossible based upon the sign patterns of the arrays. That is,

Definition (id-consistent): Sgn $\pi$ is id-consistent with sgn $\beta$ if and only if the terms being summed in (6) include at least one positive term for $\mathrm{i}=\mathrm{j}$ and at least one positive and one negative term for $\mathrm{i} \neq \mathrm{j}$.

From the perspective of the problem at issue here, this characteristic has immediate importance. 
Theorem (necessity of id-consistency): Let $\beta$ be an irreducible $\mathrm{n} x \mathrm{n}$ matrix. A proposed $\operatorname{sgn} \pi$ is a qualitative inverse of $\beta$ only if $\operatorname{sgn} \pi$ is id-consistent with $\operatorname{sgn} \beta .^{15}$

Proof. The necessity of the condition is immediate, since $\beta \pi=\mathrm{I}$ and $/$ or $\pi \beta=\mathrm{I}$ would be impossible without it. In the next section, examples show that it is not (always) sufficient."

The algorithmic principles needed to test a given $\operatorname{sgn} \pi$ for id-consistency with a $\operatorname{sgn} \beta$ are quite straight-forward and easy to apply. For example, for the entries of sgn $\beta$ proposed to be all positive, sgn $\pi$ is id-consistent if and only if each row and column of sgn $\pi$ contain at least one positive and one negative entry. Generally, no row (resp. column) of sgn $\pi$ can have exactly the same signs as the corresponding nonzero entries of any column (resp. row) of sgn $\beta$ unless $i=j$; and, no row (resp. column) of sgn $\pi$ can have exactly the negative of the signs of the corresponding nonzero entries of any column (resp. row) of sgn $\beta$. The power of this point of view for evaluating an hypothesized $\beta$ is striking. For example, if $\operatorname{sgn} \beta$ is hypothesized to be all positive, the hypothesis can be falsified by the single estimation of any row of $\pi$ and finding that the entries are all positive or all negative. Any sgn $\widehat{\boldsymbol{\pi}}$ with such a row cannot be a qualitative inverse of the hypothesized (all positive) $\beta$ regardless of the signs of its other entries. Other, similar examples can be readily constructed.

As noted, id-consistency is necessary, but not (always) sufficient. As a result, an array might be found to be id-consistent, but not found as a qualitative inverse via Monte Carlo sampling. If so, one approach would be to confront the systems (5) to see if solutions exist, or not, for the proposed $\operatorname{sgn} \beta$ and $\operatorname{sgn} \pi$. Prior to doing this, another necessary condition might be of assistance. Applied models often have a fair number of zero entries proposed for $\beta$, i.e., only a few of the roster of endogenous variables appear in any one of the equations of the system (1). Under these circumstances, although $\beta$ might not be sign non-singular, some of the arrays corresponding to its cofactors might be. If so, then some entries of $\beta$ 's adjoint can be signed, independent of magnitudes. If there are at least two such entries, then when the results of the Monte Carlo sampling is inspected, the corresponding pair of entries in $\beta^{-1}$ would have the same, or opposite, signs exactly the same number of times. Such a finding would be especially robust for the Monte

\footnotetext{
${ }^{15}$ A less formal acknowledgement of this circumstance is given in Buck and Lady (2010).
} 
Carlo approach; but as a double check, when such entries appear to be found, the symbolic expansions of the associated cofactors can be audited to confirm the circumstance. ${ }^{16}$

The condition at issue can be defined as follows,

Definition (adjoint-consistent): If $\operatorname{sgn} \mathrm{B}_{\mathrm{ij}}=\operatorname{sgn} \mathrm{B}_{\mathrm{uv}}\left(\right.$ resp. $\left.\operatorname{sgn} \mathrm{B}_{\mathrm{ij}}=-\operatorname{sgn} \mathrm{B}_{\mathrm{uv}}\right)$ independent of magnitudes, then sgn $\pi$ is adjoint-consistent with $\beta$ if and only if $\operatorname{sgn} \pi_{\mathrm{ij}}=\operatorname{sgn} \pi_{\mathrm{uv}}\left(\mathrm{resp} . \operatorname{sgn} \pi_{\mathrm{ij}}=\right.$ $\left.-\operatorname{sgn} \pi_{\mathrm{uv}}\right)$.

If applicable, this circumstance is of immediate importance.

Theorem (importance of adjoint-consistency). Let $\beta$ be an irreducible $\mathrm{n} \times \mathrm{n}$ matrix. If at least two entries of $B$ can be signed, independent of magnitudes, then a proposed $\operatorname{sgn} \pi$ is a qualitative inverse of $\beta$ only if $\operatorname{sgn} \pi$ is adjoint-consistent with $\beta$.

Proof. Necessity is immediate, since all inverses of $\beta$ must have pairs of entries that are equal or different in sign if the corresponding entries of B can be signed, independent of magnitudes. If applicable, the condition is clearly not (always) sufficient, since (say) uninvolved rows and/or columns of sgn $\pi$ may violate the requirements of id-consistency. •

As before, the implication of the condition (when applicable) is striking from the standpoint of model feasibility. Consider that if a single pair of entries of $\beta$ 's adjoint can be signed, then fully half of the possible $2^{\text {n2 }}$ sign patterns that sgn $\pi$ might take on (barring zeros) cannot be qualitative inverses of $\beta$. As a result, the proposed sgn $\beta$ could be potentially rejected due to the outcome of estimating at most two of the rows of $\pi$ as appropriate to the location of the entries at issue.

The concepts of id-consistency and adjoint-consistency, in combination with studying the system(s) (5) to see if solutions do or do not exist, completes the scope of our analysis. The

\footnotetext{
${ }^{16}$ Lady (2000) noted that this circumstance, if found, could enhance the results of using a traditional qualitative analysis.
} 
requirements of id-consistency always limits the reduced form sign patterns that can be qualitative inverses of the proposed sign pattern for the structural array. We presume and certainly hope that other conditions or algorithmic principles can be found to expedite the determination of whether or not an estimated reduced form is the qualitative inverse of a proposed structural array.

IV. Examples. The examples presented here are intended to illustrate the analytic point of view presented in the last two sections. Small arrays are used to enable the reader to confirm the results we present. Actual models would presumably almost always involve larger arrays. This is not of particular concern in testing for id-consistency, signable entries in $\beta$ 's adjoint, or testing for adjoint-consistency, since the algorithmic principles involved are well in-hand. On the other hand, determining if the systems of inequalities in (5) have solutions or not is another matter for large arrays. For the smaller arrays considered in this section, this was done by inspection and manipulation, and can be replicated by the reader.

Our first example demonstrates that even a traditional qualitative analysis can go astray, absent the expanded point of view developed in the last section. The structural array provided below was presented as an example in Lady and Maybee (1983). ${ }^{17}$

$$
\operatorname{sgn} \beta=\left[\begin{array}{llll}
- & - & 0 & - \\
+ & - & - & 0 \\
0 & + & - & - \\
+ & 0 & + & -
\end{array}\right]
$$

This matrix is sign non-singular. As a result, the entries of sgn $\beta^{-1}$ that are incident upon the nonzeros of $\beta$-transpose have the same sign as these transposed entries, independent of magnitudes. As it works out, the entries of $\operatorname{sgn} \beta^{-1}$ that are incident upon the zeros in $\beta$-transpose cannot be signed independent of magnitudes. This qualitative analysis allows sgn $\beta^{-1}$ to be written out as,

\footnotetext{
${ }^{17}$ Although sign non-singular, no full column of $\beta^{-1}$ can be signed. As a result, all of the solution values of Y cannot be signed for any specified sign pattern for Z, although some of them always can be, which was Lady and Maybee's point in presenting this array.
} 


$$
\operatorname{sgn} \beta^{-1}=\left[\begin{array}{cccc}
- & + & ? & + \\
- & - & + & ? \\
? & - & - & + \\
- & ? & - & -
\end{array}\right] .
$$

Since the four, qualitatively unsignable entries could take on a positive or negative value, there are sixteen possible outcomes for an estimated $\operatorname{sgn} \pi$ that would be consistent with the outcome of this traditional qualitative analysis; or, at least this would be the presumption of the qualitative analyst. Although Lady and Maybee didn't do this, the reader can check that all sixteen of these sign patterns are both id-consistent and adjoint-consistent. Accordingly, if the estimated reduced form takes on any of these sixteen sign patterns, then a traditional qualitative analysis would conclude that the hypothesized structural array is consistent with the data. If the estimated reduced form is not one of these sixteen sign patterns, the hypothesis is falsified.

We submitted the given structural array to Monte Carlo sampling and after taking a fair number of samples, i.e., millions, we found that two of the sixteen sign patterns that (seemingly) $\beta^{-1}$ might take on never showed up as qualitative inverses. These two arrays are given below: ${ }^{18}$

$$
\begin{gathered}
\text { (sign pattern \#21392): } \operatorname{sgn} \beta^{-1}=\left[\begin{array}{cccc}
- & + & -* & + \\
- & - & + & +* \\
+* & - & - & + \\
- & -* & - & -
\end{array}\right] ; \text { and, } \\
\left(\text { sign pattern \#29204): } \operatorname{sgn} \beta^{-1}=\left[\begin{array}{cccc}
- & + & +* & + \\
- & - & + & -* \\
-* & - & - & + \\
- & +* & - & -
\end{array}\right] .\right.
\end{gathered}
$$

\footnotetext{
${ }^{18}$ Indices are assigned to the possible sign patterns that $\beta^{-1}$ might take by first constructing the binary number that corresponds to the rows of the array, first to last, written out as a sixteen digit binary number with " 0 " corresponding to "-. and " 1 " corresponding to "+."The index is then computed as the corresponding base 10 number. E.g., an all negative array has an index of " 0 " and an all positive array has an index of "65535."
} 
The four variable signs at issue are indicated with an "*." We wrote out the systems (5) corresponding to both of these sign patterns and found them to be impossible, i.e., assuming there to be a solution resulted in a logical inconsistency. This derivation is provided in the appendix. As a result, even in the rare case that a traditional qualitative analysis could reach conclusions about signable entries in $\beta^{-1}$, the configuration of generally unsignable entries that might still remain would not be critically assessed. As a result, for such as the case above, an estimated reduced form sign pattern might be accepted as consistent with the data, although in fact the sign pattern found was not a qualitative inverse of the hypothesized structural array. Without the further analysis of the possible sign patterns for the unsignable entries, the traditional qualitative analysis would have missed the very circumstance it was intended to detect.

The second example is designed to capture all of the features of the analysis outlined in the last section. The assumed hypothesized array is given below,

$$
\operatorname{sgn} \beta=\left[\begin{array}{llll}
- & + & + & 0 \\
+ & - & + & + \\
+ & + & - & + \\
0 & + & + & -
\end{array}\right] .
$$

This sign pattern, negative main diagonal entries and non-negative off diagonal entries, is an example of a Metzler (1945) matrix, a form corresponding to the excess demand functions for multimarket equilibria for which all commodities are (weakly, if zeros are allowed) gross substitutes. Since all off-diagonal entries are non-negative, all cycles of the corresponding $\operatorname{SDG}(\beta)$ must have positive values and the array is not sign non-singular. I.e., no entry of $\beta^{-1}$ can be signed, independent of magnitudes. Accordingly, the conditions for a successful, traditional qualitative analysis are not satisfied.

In principle, there are 65536 sign patterns (barring zeros) that a 4 x 4 array can take on. Each of these was tested for id-consistency with the hypothesized $\beta$. Only 229 of these sign patterns satisfied the conditions. Accordingly, over $99.6 \%$ of the potential outcomes for an estimated $\pi$ 
cannot be qualitative inverses of the hypothesized $\beta$, a dramatic example of the limitations imposed on the reduced form by a specification of the sign pattern of the structural array.

Repeated samples of the Monte Carlo further reduced the set of possible $\pi$ and found only 110 sign patterns for $\beta^{-1}$. This left 119 sign patterns that were id-consistent, but potentially proposing systems of inequalities (5) that did not have solutions. The Monte Carlo sampling also reported that four entries of the simulated $\beta^{-1}$ always had the same sign. The symbolic expansions of the associated cofactors were written out and these terms were all found to be negative, independent of magnitudes. Hence, the sign pattern of $\beta$ 's adjoint could be shown to be,

$$
\operatorname{sgn} \operatorname{Adjoint}(\beta)=\left[\begin{array}{cccc}
? & ? & ? & - \\
? & ? & - & ? \\
? & - & ? & ? \\
- & ? & ? & ?
\end{array}\right]
$$

Each of the 229 id-consistent sign patterns was additionally tested for adjoint-consistency. Of these, 112 were not adjoint-consistent leaving 117 sign patterns that satisfied the two necessary conditions. Of these, seven were not found by Monte Carlo sampling to be qualitative inverses of the hypothesized array. These sign patterns are given below. Given knowledge of the signable entries of the adjoint, the sign of the determinant is also given.

$$
\begin{aligned}
& \left(\operatorname{sign} \text { pattern \#51): } \operatorname{sgn} \beta^{-1}=\left[\begin{array}{llll}
- & - & - & - \\
- & - & - & - \\
- & - & + & + \\
- & - & + & +
\end{array}\right] ; \text { and, det } \beta>0 .\right. \\
& \left(\operatorname{sign} \text { pattern \#1285) : } \operatorname{sgn} \beta^{-1}=\left[\begin{array}{llll}
- & - & - & - \\
- & + & - & + \\
- & - & - & - \\
- & + & - & +
\end{array}\right] ; \text { and, det } \beta>0 .\right.
\end{aligned}
$$




$$
\begin{aligned}
& \text { (sign pattern \#41120): } \operatorname{sgn} \beta^{-1}=\left[\begin{array}{llll}
+ & - & + & - \\
- & - & - & - \\
+ & - & + & - \\
- & - & - & -
\end{array}\right] ; \text { and, det } \beta>0 \text {. } \\
& \left(\text { sign pattern \#44085): } \operatorname{sgn} \beta^{-1}=\left[\begin{array}{llll}
+ & - & + & - \\
+ & + & - & - \\
- & - & + & + \\
- & + & - & +
\end{array}\right] ; \text { and, det } \beta>0\right. \text {. } \\
& \text { (sign pattern \#50595): } \operatorname{sgn} \beta^{-1}=\left[\begin{array}{llll}
+ & + & - & - \\
- & + & - & + \\
+ & - & + & - \\
- & - & + & +
\end{array}\right] ; \text { and, det } \beta>0 . \\
& \text { (sign pattern \#52224): } \operatorname{sgn} \beta^{-1}=\left[\begin{array}{llll}
+ & + & - & - \\
+ & + & - & - \\
- & - & - & - \\
- & - & - & -
\end{array}\right] ; \text { and, } \operatorname{det} \beta>0 \text {. } \\
& \text { ( sign pattern \#64479) : } \operatorname{sgn} \beta^{-1}=\left[\begin{array}{llll}
+ & + & + & + \\
+ & - & + & + \\
+ & + & - & + \\
+ & + & + & +
\end{array}\right] \text {; and, det } \beta<0 .
\end{aligned}
$$

Each of these was assessed with respect to the systems of inequalities (5) and found to be impossible (derivations are given in the appendix). Accordingly, the sample(s) used for the Monte Carlo were sufficiently large.

This example shows the substantial limitations that the structural sign pattern can impose upon the sign pattern of the reduced form. The conditions of id-consistency always apply and demonstrate that a specification of the sign pattern of the structural array always imposes limits 
on the corresponding reduced form. Adjoint-consistency may not always apply, but it is potentially applicable for structural arrays with a fair number of zero entries. Given an estimated reduced form that satisfies the two necessary conditions, but is not found by Monte Carlo sampling, an assessment of the existence of the corresponding system(s) of inequalities (5) remains an option in terms of validating the hypothesized structural sign pattern.

V. Summary. Over the course of recent decades, economists have proceeded by specifying systems of equations designed to express some feature of how the economy works or explain the behavior of its embodied decision makers, such termed as the structural model. The relationships among the endogenous and exogenous variables of the structural model are then brought to the data by estimating the (usually) unrestricted reduced form. In computing estimates of the structural unknowns the empiricist applies the data to the reduced form, while ignoring the restrictions implied by the structure, then works back to the structural unknowns. The research effort then proceeds to a discussion of statistically significant estimates of the structural unknowns, all the while being ignorant of or ignoring the fact that the model may have been falsified by the realization of a reduced form that could not have occurred if the original model had been correct. ${ }^{19}$

Having a methodology at hand for detecting when a proposed model could not possibly have generated the observed data is essential. The argument presented here is that the sign pattern of the proposed structural model always restricts the set of permissible sign patterns for the directly estimable reduced form. In the event that an observed reduced form is not a member of the set of permissible reduced forms then the model has been falsified.

The method for enumerating the set of permissible reduced forms has been developed here. Two theorems stating the necessary conditions for the enumeration of permissible reduced are stated and proved. The first necessary condition, the id-condition or the identity condition, exploits the fact that the product, from the left and the right, of a matrix and its inverse must be the identity matrix. The second necessary condition, adjoint-consistency, exploits the fact that a proposed

\footnotetext{
${ }^{19}$ Estimates of unknowns can be statistically significant because the estimated unknowns are, after all, (conditional and/or partial) correlations between pairs of variables. Such correlations can be significant regardless of whether the correct underlying data generating process has been specified.
} 
inverse must be consistent with (if at least two) signable entries in its adjoint. These two necessary conditions considerably restrict the number of conceivable inverses of $\beta$. It is also shown the set of candidate inverses can be further reduced by finding that proposed systems of inequalities (5) are impossible, i.e., algebraic methods are used (in the Appendix) to show that the sign patterns not found by the Monte Carlo as qualitative inverses result in logical inconsistencies. The line of reasoning and method are illustrated with some simple examples.

\section{References}

Awokuse, Titus O. and David A. Bessler, 2003, "Vector Autoregressions, Policy Analysis, and Directed Acyclic Graphs: An Application to the U.S. Economy," Journal of Applied Economics, 6(1), pp. 1-24.

Bassett, Lowell, John Maybee, and James Quirk, 1968, "Qualitative Economics and the Scope of the Correspondence Principal," Econometrica, 36, pp. 544-563.

Buck, Andrew and George Lady, 2005, "Falsifying Economics Models," Economic Modelling, 22, September 2005, pp. 777-810. , 2010, “An Expanded Scope For Qualitative Economics,” Temple University Department of Economics Working Paper, DETU 10-07.

Gillen, W.J. and A. Guccione, (1990), “The Introduction of Constraints in Lancaster's Qualitative Comparative Statics Algorithm," Quarterly Journal of Economics, 105, 1053-1061.

Gorman, Terrance, (1964), "More Scope for Qualitative Economics," Review of Economic Studies, 31, pp. 65-68.

Hale, Douglas, George Lady, John Maybee, and James Quirk, 1999, Nonparametric Comparative Statics and Stability, Princeton, New Jersey: Princeton University Press.

Hicks, J.R., (1932), The Theory of Wages, London, Macmillan. Horn, Roger and Charles Johnson, (1985), Matrix Analysis, Cambridge University Press. 
Lancaster, Kelvin, 1962, "The Scope of Qualitative Economics," Review of Economic Studies, 29, pp. 99-132.

, 1966, "The Solution of Qualitative Comparative Statics Problems," Quarterly Journal of Economics, 53, 278-295.

Lady, George, 1983, "The Structure of Qualitatively Determinate Relationships," Econometrica, 51, pp. 197-218.

,2000, “Topics in Nonparametric Comparative Statics and Stability," International Advances in Economic Research, 6, pp. 67-83.

Lady, George and John Maybee (1983), "Qualitatively Invertible Matrices," Mathematical Social Sciences, 6, 397-407.

Lady, George and James Quirk, 2007, "The Scope of the LeChatelier Principle," Physica A:

Statistical Mechanics and its Applications, 381, 351-365. , 2010, “The Global LeChatelier Principle and Multimarket

Equilibria," The Review of Economic Design, 14, 1, pp. 193-201.

Maybee, John, 1966, "New Generalizations of Jacobi Matrices", SIAM Journal of Applied Mathematics, Vol. 14.

Maybee, John and James Quirk, 1969, “Qualitative Problems in Matrix Theory,” SIAM Review, $11,30-51$.

Maybee, John and Garry Weiner, 1988, "From Qualitative Matrices to Quantitative Restrictions," Linear and Multilinear Algebra, 22, 229-248. 
Metzler, Llyod, (1945), "Stability of Multiple Markets: The Hicks Conditions," Econometrica, $13,277-292$.

Popper, Karl , [1934] 1959, The Logic of Scientific Discovery, reprint, New York: Harper and Row.

Ritschard, Gilbert, (1983), “Computable Qualitative Comparative Static Techniques," Econometrica, 51(4), 1145- 1168.

Samuelson, Paul, (1947), Foundations of Economic Analysis, Cambridge: Harvard University Press. ,1960, “An Extension of the LeChatelier Principle,” Econometrica, 28, 368-

379.

\section{Appendix}

It is useful to set up the graph theoretic organization of $\beta$ for the purpose of considering the systems (5) corresponding to the examples in section IV. The array $\beta$ is first arranged such that $\beta_{\mathrm{ii}}<0$ for all $\mathrm{i}$. This amounts to placing one of the nonzero terms in the expansion of $\beta$ 's determinant on its main diagonal and changing the signs of (say) rows as necessary. So arranged, the matrix is said to be in "standard form." The qualitatively decidable attributes of $\beta$ are not changed by these manipulations. Next, mark and enumerate places on the page, one for each variable, i.e., column of $\beta$. The places marked are called "vertices." Draw arrows among the vertices following the convention:

$$
(\mathrm{j}) \rightarrow(\mathrm{i}) \text { if and only if } \beta_{\mathrm{ij}} \neq 0 \text {. }^{20}
$$

Place a "+" or "_" subscripting at the head of each arrow as appropriate to the sign of the nonzero entry of $\beta$ to which it corresponds, e.g.,

${ }^{20}$ The transposed convention is often used, i.e., (i ) $\rightarrow(j)$ if and only if $\beta_{\mathrm{ij}} \neq 0$, and some of the literature cited here follows this convention. This would be entirely intuitive for $\beta_{\mathrm{ij}}$ (such as) the technological coefficient in an inputoutput model identifying the flow of a good or service from sector \#i to \#j. The convention here is used to highlight that the entry $\beta_{\mathrm{ij}}$ represents a flow of inference from variable $\# \mathrm{j}$ to $\# \mathrm{i}$ for $\beta$ in standard form. 


$$
(\mathrm{j}) \rightarrow_{+}(\mathrm{i}) \text { if and only if } \beta_{\mathrm{ij}}>0 \text {. }
$$

The arrows are called "signed directed arcs" and the entire array of vertices and signed arrows is called the "signed directed graph $(\operatorname{SDG}(\beta))$ " corresponding to $\beta$ (as transformed). A traversal of the $\operatorname{SDG}(\beta)$ by following the arrows from one vertex to another without passing through an intermediate vertex more than once is called a "path." A traversal from a vertex to itself without passing through an intermediate vertex more than once is called a "cycle." Cycles are "disjoint" if they share no vertices in common. The signs and values of cycles and paths are determined by the signs and values of the products of the entries of $\beta$ that correspond to their embodied signed, directed arcs.

The usefulness of this arrangement is that it can be shown that each term in the expansion of det $\beta$, other than the product of the main diagonal entries, is composed of the products of the values of some number of disjoint cycles with main diagonal entries included to account for vertices not present in the embodied cycles. ${ }^{21}$ The entire expansion of det $\beta$ is the sum of the product of the main diagonal entries and all possible terms comprised of distinct combinations of disjoint cycles and main diagonal entries. Given this, it is known additionally for $\beta$ in standard form that for terms that embody only negative cycles, the sign of the term is $(-1)^{\mathrm{n}}$ which is also the sign of the product of the main diagonal entries. Terms that embody an even number of positive cycles also have this sign. It is only terms that embody an odd number of positive cycles that have the opposite sign, $(-1)^{\mathrm{n}-1}$. These circumstances provide the fundamental theorem of traditional qualitative analysis, as provided by Bassett, Maybee and Quirk (1968); namely, all terms in the expansion of det $\beta$ have the same sign (which is $(-1)^{\mathrm{n}}$ for $\beta$ in standard form) if and only if all cycles in $\operatorname{SDG}(\beta)$ have negative values, i.e., $\beta$ is sign non-singular.

It is immediate that $\beta^{-1}$ can have entries with particular signs independent of the magnitudes of the entries of $\beta$ if and only if $\beta$ is sign non-singular. For $\beta$ sign non-singular, $\operatorname{sgn}\left(\beta^{-1}\right)_{i j}$ has a

\footnotetext{
${ }^{21}$ Strictly speaking the main diagonal entries can be considered to correspond to cycles of length "one," so the term comprised of their product is consistent with the convention used to express the other terms.
} 
particular sign independent of the magnitudes of the entries of $\beta$ if and only if all paths from vertex $\# \mathrm{j}$ to vertex $\# \mathrm{i}$ have the same sign. For $\beta$ sign non-singular, this must be true for $\beta_{\mathrm{ji}} \neq 0$ and may or may not be true for $\beta_{\mathrm{ji}}=0$. If all paths from vertex $\# \mathrm{j}$ to vertex \#i have the same sign, then $\operatorname{sgn} \beta_{\mathrm{ij}}{ }^{-1}$ is the negative of that sign, independent of the magnitudes of the entries of $\beta{ }^{22}$ Wewill use these graph theoretic properties of $\beta$ in working through the examples that follow.

The first example in section IV considered the structural hypothesis given by,

$$
\operatorname{sgn} \beta=\left[\begin{array}{cccc}
- & - & 0 & - \\
+ & - & - & 0 \\
0 & + & - & - \\
+ & 0 & + & -
\end{array}\right] .
$$

This array is sign non-singular and twelve of the sixteen entries of its inverse can be signed using the graph theoretic results cited above, independent of magnitudes, as given below,

$$
\operatorname{sgn} \beta^{-1}=\left[\begin{array}{cccc}
- & + & ? & + \\
- & - & + & ? \\
? & - & - & + \\
- & ? & - & -
\end{array}\right] .
$$

Since the four entries marked "?" can each be positive or negative, it would be traditionally supposed that there would be sixteen reduced form sign patterns that, if estimated, would be consistent with the data, i.e., those sign patterns consistent with the twelve signable entries, regardless of the other four signs. In general, this supposition presumes that the four unsignable entries can take on signs independent of each other. Our point is that this need not be true. For this case it is not, and two of the sixteen sign patterns for the four unsignable entries are not possible for inverses of the proposed structural array, as given below,

\footnotetext{
${ }^{22}$ See also Maybee and Quirk (1969).
} 


$$
\begin{aligned}
\left(\operatorname{sign} \text { pattern \#21392): } \operatorname{sgn} \beta^{-1}=\left[\begin{array}{cccc}
- & + & -* & + \\
- & - & + & +* \\
+* & - & - & + \\
- & -* & - & -
\end{array}\right] ; \text { and },\right. \\
\left(\operatorname{sign} \text { pattern \#29204): } \operatorname{sgn} \beta^{-1}=\left[\begin{array}{cccc}
- & + & +* & + \\
- & - & + & -* \\
-* & - & - & + \\
- & +* & - & -
\end{array}\right] .\right.
\end{aligned}
$$

The suspicion that these sign patterns were not qualitative inverses of the proposed structural array arose since they were repeatedly not found by the Monte Carlo for very large samples. Still, both of these arrays are id-consistent and adjoint-consistent with the proposed sgn $\beta$. Accordingly, to show that these two sign patterns are not possible qualitative inverses requires that it be shown that the system(s) of inequalities (5) do not have solutions for these proposed sign patterns.

Since the determinant and twelve entries of $\operatorname{sgn} \beta^{-1}$ are all signable for these cases, it is sufficient to inspect the expansions of the four cofactors corresponding to the unsignable entries. In writing out these expansions a shortcut that can sometimes ease the burden of derivation is based upon early work by Maybee (1966). Here it was shown that each term in the expansion of the (j,i)th cofactor, i.e., the (i,j)th entry of the adjoint, can be expressed by,

$$
\mathrm{v}(\operatorname{path}(\mathrm{j} \rightarrow \mathrm{i}))(-1)^{\mathrm{q}} \beta(\operatorname{path}(\mathrm{j} \rightarrow \mathrm{i}))
$$

where $v($ path $(j \rightarrow i))$ is the product of the entries of $\beta$ that correspond to the directed arcs of the path, $q$ is the length of the path (i.e., the number of embodied directed $\operatorname{arcs})$, and $\beta(\operatorname{path}(j \rightarrow i))$ is the principle minor of $\beta$ corresponding to the array formed by deleting the rows and columns that correspond to the vertices in the path. Given this, the terms (there are two) in the expansion of (say, the 1,3 term in the adjoint of $\beta$ ) $\mathrm{B}_{13}$ are given by (with the hypothesized signs of the entries given in parentheses): 


$$
\mathrm{B}_{13}=\left(\beta_{43}(+) \beta_{14}(-) \beta_{22}(-)\right)+\left(\beta_{23}(-) \beta_{12}(-) \beta_{44}(-)\right) .
$$

The issue at stake is to determine if this expansion, and the other three, collectively have a solution if set to the sign pattern proposed for $\beta$ 's adjoint. Based on the Monte Carlo results the expectation of the derivation is that they do not.

To ease the burden of derivation further, $\beta$ will be considered to have its main diagonal normalized such that $\beta_{\mathrm{ii}}=-1$ for all $\mathrm{i}$. This can be accomplished by (say) multiplying each column of $\beta$ by the absolute value of the reciprocal of its main diagonal entry. So formed, the amended array has the same qualitative properties as $\beta$. Finally, the expansions will be expressed in terms of the absolute values of $\beta$ 's entries with advocated signs accounted for in the way the sum is formed. Given this, the system of inequalities is expressed below. The expansions are set to the sign pattern advocated for array \#21392. In the derivations below $a_{i j}=\operatorname{abs}\left(\beta_{i j}\right)$.

$$
\begin{aligned}
& \text { (A1) for } \mathrm{B}_{13}:\left(\mathrm{a}_{43} \mathrm{a}_{14}\right)-\left(\mathrm{a}_{23} \mathrm{a}_{12}\right)<0 \\
& \text { (A2) for } \mathrm{B}_{24}:\left(\mathrm{a}_{14} \mathrm{a}_{21}\right)-\left(\mathrm{a}_{34} \mathrm{a}_{23}\right)>0 \text {; } \\
& \text { (A3) for } \mathrm{B}_{31}:\left(\mathrm{a}_{41} \mathrm{a}_{34}\right)-\left(\mathrm{a}_{21} \mathrm{a}_{32}\right)>0 \\
& \text { (A4) for } \mathrm{B}_{42}:\left(\mathrm{a}_{12} \mathrm{a}_{41}\right)-\left(\mathrm{a}_{32} \mathrm{a}_{43}\right)<0 \text {. }
\end{aligned}
$$

Assume a solution exists. Then, manipulating (A1) and (A4):

$$
\mathrm{a}_{43}<\left(\mathrm{a}_{23} \mathrm{a}_{12}\right) / \mathrm{a}_{14} ; \text { and, } \mathrm{a}_{43}>\left(\mathrm{a}_{12} \mathrm{a}_{41}\right) / \mathrm{a}_{32} .
$$

Combine these results, divide by $\mathrm{a}_{12}$ and rearrange,

$$
\left.\left(a_{23} a_{32}\right)>\left(a_{14} a_{41}\right) \text {. (Condition } U\right) \text {. }
$$

Now, manipulating (A2) and (A3):

$$
\mathrm{a}_{34}<\left(\mathrm{a}_{14} \mathrm{a}_{21}\right) / \mathrm{a}_{23} ; \text { and, } \mathrm{a}_{34}>\left(\mathrm{a}_{21} \mathrm{a}_{32}\right) / \mathrm{a}_{41} .
$$


Combine these results, divide by $\mathrm{a}_{21}$ and rearrange,

$$
\left.\left(\mathrm{a}_{14} \mathrm{a}_{41}\right)>\left(\mathrm{a}_{23} \mathrm{a}_{32}\right) \text {. (Condition } \mathrm{V}\right) \text {. }
$$

Condition $\mathrm{U}$ and Condition $\mathrm{V}$ are jointly impossible. Accordingly, the system of four inequalities does not have a solution and sign pattern \#21392 cannot be a qualitative inverse of the proposed structural sign pattern. All four inequalities are reversed for sign pattern \#29204 and a similar contradiction would be found. The Monte Carlo results are vindicated analytically.

The second example in section IV above considered the structural hypothesis given by,

$$
\operatorname{sgn} \beta=\left[\begin{array}{cccc}
- & + & + & 0 \\
+ & - & + & + \\
+ & + & - & + \\
0 & + & + & -
\end{array}\right]
$$

Of the possible $655364 \times 4$ sign patterns (barring zeros) eligible to be qualitative inverses of this array, only 117 satisfied the two necessary conditions: id-consistency and (which applies to this case) adjoint-consistency. Of these, only 110 were found by the Monte Carlo. The seven that were not found are enumerated above in section IV. All of these were assessed with respect to solutions to the corresponding systems of inequalities (5) and found to be impossible, validating the findings of the Monte Carlo. The derivations for the two most difficult cases are given below.

The sign patterns for the two (that we found to be most difficult) cases are:

$$
\text { ( sign pattern \#44085) : } \operatorname{sgn} \beta^{-1}=\left[\begin{array}{cccc}
+ & - & + & - \\
+ & + & - & - \\
- & - & + & + \\
- & + & - & +
\end{array}\right] \text {; and, det } \beta>0 \text {. }
$$




$$
\text { (sign pattern \#50595): } \operatorname{sgn} \beta^{-1}=\left[\begin{array}{llll}
+ & + & - & - \\
- & + & - & + \\
+ & - & + & - \\
- & - & + & +
\end{array}\right] \text {; and, det } \beta>0 \text {. }
$$

Rather than undertake an analysis of the system of seventeen inequalities spelled out in (5), the Monte Carlo sampling was first used see if some subset of signed entries in these arrays failed to appear, regardless of the signs of other entries. If so, attention could be directed towards this smaller number of inequalities. As it worked out, the following two partial sign patters were not found, regardless of the signs for entries marked "*."

$$
\begin{aligned}
& \operatorname{sgn} \beta^{-1}=\left[\begin{array}{llll}
* & - & * & * \\
+ & * & * & * \\
* & * & * & * \\
- & * & * & *
\end{array}\right] ; \text { and, } \operatorname{det} \beta>0 . \\
& \operatorname{sgn} \beta^{-1}=\left[\begin{array}{llll}
* & + & * & * \\
- & * & * & * \\
* & * & * & * \\
- & * & * & *
\end{array}\right] ; \text { and, } \operatorname{det} \beta>0 .{ }^{23}
\end{aligned}
$$

Since $\mathrm{B}_{41}<0$, it must be that det $\beta>0$ for both arrays. In addition, the pair of given signs are the negative of each other across the two arrays. This then presents the following two inequalities for analysis:

$$
\begin{aligned}
& \text { (A5) } \operatorname{det} \beta>0 \\
& \text { (A6) } B_{12} B_{21}<0
\end{aligned}
$$

The graph theoretic organization of $\beta$ outlined above will be used in the derivations. As before, $\beta$ has been manipulated such that $\beta_{\mathrm{ii}}=-1$ for all $\mathrm{i}$. Inspection reveals that there are eleven cycles in $\operatorname{SDG}(\beta)$. These are enumerated below (all paths and cycles are positive).

\footnotetext{
${ }^{23}$ Admittedly, there was some trial and error in this finding. Still, as immediately noted below, since all cycles are positive for the hypothesized array, certain combinations of signs seem less plausible than others.
} 


$\begin{array}{lll}\text { Cycle \# } & \text { Directed Graph } & \text { Value } \\ \text { C1 } & 1 \rightarrow 2 \rightarrow 1 & \beta_{21} \beta_{12} \\ \text { C2 } & 1 \rightarrow 2 \rightarrow 3 \rightarrow 1 & \beta_{21} \beta_{32} \beta_{13} \\ \text { C3 } & 1 \rightarrow 2 \rightarrow 4 \rightarrow 3 \rightarrow 1 & \beta_{21} \beta_{42} \beta_{34} \beta_{13} \\ \text { C4 } & 1 \rightarrow 3 \rightarrow 1 & \beta_{31} \beta_{13} \\ \text { C5 } & 1 \rightarrow 3 \rightarrow 2 \rightarrow 1 & \beta_{31} \beta_{23} \beta_{12} \\ \text { C6 } & 1 \rightarrow 3 \rightarrow 4 \rightarrow 2 \rightarrow 1 & \beta_{31} \beta_{43} \beta_{24} \beta_{12} \\ \text { C7 } & 2 \rightarrow 3 \rightarrow 2 & \beta_{32} \beta_{23} \\ \text { C8 } & 2 \rightarrow 3 \rightarrow 4 \rightarrow 2 & \beta_{32} \beta_{43} \beta_{24} \\ \text { C9 } & 2 \rightarrow 4 \rightarrow 2 & \beta_{42} \beta_{24} \\ \text { C10 } & 2 \rightarrow 4 \rightarrow 3 \rightarrow 2 & \beta_{42} \beta_{34} \beta_{23} \\ \text { C11 } & 3 \rightarrow 4 \rightarrow 3 & \beta_{43} \beta_{34}\end{array}$

Inspection further reveals that there are two pairs of disjoint cycles: $\{\mathrm{C} 1$ and $\mathrm{C} 11\}$ and $\{\mathrm{C} 4$ and C9\}. Accordingly, (A5) can be written out as,

$$
\text { (A5) det } \beta=1+C 1 C 11+C 4 C 9-\sum_{k=1}^{11} C_{k}>0 .
$$

For $\mathrm{B}_{12}$ and $\mathrm{B}_{21}$ inspection reveals that there are four distinct paths, i.e., four terms, in the expansion of each of the corresponding cofactors. As a preliminary finding, multiplying through by the "return" path for each cofactor expresses the results in terms of cycles and establishes the size of C11; namely,

$$
\mathrm{B}_{12} \beta_{21}=-\mathrm{C} 1+\mathrm{C} 1 \mathrm{C} 11-\mathrm{C} 2-\mathrm{C} 3>0 \text { for } \# 50595 \text {. }
$$

For this inequality to hold, it must be that $\mathrm{C} 11>1$. And also,

$$
\mathrm{B}_{21} \beta_{12}=-\mathrm{C} 1+\mathrm{C} 1 \mathrm{C} 11-\mathrm{C} 5-\mathrm{C} 6>0 \text { for } \# 44085 \text {. }
$$

As before, for this inequality to hold, it must be that $\mathrm{C} 11>1$.

Since the paths in each cofactor are, so to speak, running in opposite directions, the products of the expansions of these two cofactors are (conveniently) also expressible in terms of cycles. It is a bit laborious, but the product of these two cofactors can be written out as,

(A6) $\mathrm{B}_{12} \mathrm{~B}_{21}=\mathrm{Q} 1+\mathrm{Q} 2+\mathrm{Q} 3+\mathrm{Q} 4<0$, where,

$$
\mathrm{Q} 1=\mathrm{C} 1-\mathrm{C} 1 \mathrm{C} 11+\mathrm{C} 5+\mathrm{C} 6
$$




$$
\begin{gathered}
\mathrm{Q} 2=-\mathrm{C} 1 \mathrm{C} 11+\mathrm{C} 1 \mathrm{C} 11^{2}-\mathrm{C} 5 \mathrm{C} 11-\mathrm{C} 6 \mathrm{C} 11 ; \\
\mathrm{Q} 3=\mathrm{C} 2-\mathrm{C} 2 \mathrm{C} 11+\mathrm{C} 4 \mathrm{C} 7+\mathrm{C} 4 \mathrm{C} 8 ; \text { and, finally, } \\
\mathrm{Q} 4=\mathrm{C} 3-\mathrm{C} 3 \mathrm{C} 11+\mathrm{C} 4 \mathrm{C} 10+\mathrm{C} 4 \mathrm{C} 9 \mathrm{C} 11 .
\end{gathered}
$$

Before combining these expressions, some convenient arrangements can be made. The first two terms in each of Q1 and Q2 can be written as,

$$
\mathrm{C} 1-\mathrm{C} 1 \mathrm{C} 11-\mathrm{C} 1 \mathrm{C} 11+\mathrm{C} 1 \mathrm{C} 11^{2}=\mathrm{C} 1(1-\mathrm{C} 11)^{2} .
$$

There are two terms involving each of C2, C3, C5, and C6 that can be organized as

$$
\mathrm{C}_{\mathrm{i}}(1-\mathrm{C} 11), \mathrm{i}=2,3,5,6 .
$$

There are three terms involving $\mathrm{C} 4$ which can be written as $\mathrm{C} 4(\mathrm{C} 7+\mathrm{C} 8+\mathrm{C} 10)$ and the last term is $\mathrm{C} 4 \mathrm{C} 9 \mathrm{C} 11$.

This term can be rewritten as: C4C9C11 = C4C9 - (1 - C11)C4C9.

Given this, assemble the terms as follows:

$$
\mathrm{X}=(\mathrm{C} 1-\mathrm{C} 1 \mathrm{C} 11+\mathrm{C} 2+\mathrm{C} 3+\mathrm{C} 5+\mathrm{C} 6-\mathrm{C} 4 \mathrm{C} 9)
$$

Now, (A6) can be expressed based on the above,

$$
\mathrm{B}_{12} \mathrm{~B}_{21}=(1-\mathrm{C} 11) \mathrm{X}+\mathrm{C} 4(\mathrm{C} 7+\mathrm{C} 8+\mathrm{C} 10)+\mathrm{C} 4 \mathrm{C} 9<0 .
$$

Since from the above, $\mathrm{C} 11>1, \mathrm{X}>0$ is necessary for the inequality (A6) to hold. Given this, consider that:

$$
\operatorname{det} \beta+X=1-C 4-\sum_{i=7}^{11} C_{i}>0 .
$$

Since also, C11-1>0, this gives, 


$$
\operatorname{det} \beta+X+C 11-1=-C 4-\sum_{i=7}^{10} C_{i}>0,
$$

which is impossible, since all cycles have positive values. Thus, the Monte Carlo is vindicated for the two sign patterns, \#44085 and \#50595: they cannot be qualitative inverses. The derivations for the other five sign patterns not found also can be expressed in terms of conditions of inequalities involving cycles and show that the sign patterns could not be qualitative inverses. The derivations are somewhat easier than the above. 